\title{
An Extension of Fuzzy Relational Compositions Using Generalized Quantifiers
}

\author{
Nhung Cao ${ }^{1}$ Martin Štěpnička ${ }^{1}$ Michal Holčapek ${ }^{1}$ \\ ${ }^{1}$ Institute for Research and Applications of Fuzzy Modeling, CE IT4Innovations, University of Ostrava \\ 30. dubna 22, 70103 Ostrava, Czech Republic \\ nhung.cao@osu.cz, martin.stepnicka@osu.cz, michal.holcapek@osu.cz
}

\begin{abstract}
Fuzzy relational compositions have been extensively studied by many authors. Especially, we would like to express studies of the fuzzy relational compositions motivated by their applications to medical diagnosis by Willis Bandler and Ladislav Kohout. These types of compositions use only two quantifiers: universal quantifier and existential quantifier. There exists a huge gap between these quantifiers. This is a natural motivation for studying fuzzy relational compositions defined based on generalized quantifiers. In this paper, we revisit and provide definitions of these compositions as well as those of derived images and preimages. Furthermore, we investigate their mathematical properties.
\end{abstract}

Keywords: Fuzzy relations, Sup-T composition, Inf-R composition, Bandler-Kohout products.

\section{Introduction}

Fuzzy relational compositions provide an extension of classical relational compositions and have been firstly studied by Willis Bandler and Ladislav Kohout between late 70's and early 80's. Later on numerous researchers deeply elaborated various aspects of fuzzy relation compositions (see, e.g., $[1,2]$ and references therein). Fuzzy relational compositions have an important role in many areas of fuzzy mathematics, including the formal constructions of fuzzy inference systems $[3,4,5,6]$, medical diagnosis [7], architectures of information processing [8] or in flexible queries to relational databases $[9,10,11]$.

This paper is a continuation of our research in the field of fuzzy relational compositions started in [12], where we introduced fuzzy relational compositions with an application of generalized (fuzzy) quantifier of type $\langle 1\rangle$ determined by fuzzy measures (see [13]) and showed several basic properties. The paper is organized as follows.

Section 2 recalls four classical relational compositions that motivated by their application to medical diagnosis then naturally extend to fuzzy relational compositions using suitable operations which involves t-norms and residual implications.

Section 3 formally deals with fuzzy relational compositions based on generalized quantifiers. This is motivated by the fact that standard compositions implicitly employ the existential quantifier (where just one element is enough to result the truth) or the universal quantifier (where all elements have to fulfill a given formula in order to result the truth). Thus, there exists a huge gap between these quantifiers and investigation of compositions based on generalized quantifiers is necessary. In this paper, we consider the quantifiers such as "Most", "Many", or "A few" and of a similar type.

Section 4 introduces properties of such newly defined fuzzy relational compositions. We show, that some of the well-known properties of fuzzy relational compositions are preserved only in weak form, in the case of the compositions based on generalized quantifiers.

Finally, Section 5 introduces definitions and properties of images and preimages of an arbitrary fuzzy set under fuzzy relations using generalized quantifiers.

\section{Relational compositions and fuzzy relational compositions}

\subsection{Relational compositions}

Let us consider three non-empty finite universes $X, Y$, and $Z$. Following the work of Willis Bandler and Ladislav Kohout, for the sake of illustrative nature, we can assume that $X$ is a finite set of patients, $Y$ is a finite set of symptoms, $Z$ is a finite set of diseases.

Let $R$ be a relation on $X \times Y$ and $S$ be a relation on $Y \times Z$. For the purpose of the paper we assume that $(x, y) \in R$ means that a patient $x$ has a symptom $y$ and similarly, $(y, z) \in S$ means that a symptom $y$ belongs to a disease $z$. Both relations are usually at disposal because $R$ can be easily obtained by asking the patient or by measuring the symptom, and $S$ can be easily found from medical literature. The usual diagnosis task of a physician is to state what are the potential dis- 
eases of a given patient. From the mathematical point of view, the desired relationship between patients and diseases is a suitable composition of the relations $R$ and $S$. We may define a binary relation on $X \times Z$ using a composition @ based on the following scheme:

$$
\begin{array}{ccccccc}
R & \subseteq & X & \times & Y & & \\
S & \subseteq & & & Y & \times & Z \\
\hline R @ S & \subseteq & X & & & \times & Z .
\end{array}
$$

Four fundamental compositions are given as follows

$$
\begin{aligned}
R \circ S= & \{(x, z) \in X \times Z \mid \\
& \exists y \in Y:(x, y) \in R \&(y, z) \in S\}, \\
R \triangleleft S= & \{(x, z) \in X \times Z \mid \\
& \forall y \in Y:(x, y) \in R \Rightarrow(y, z) \in S\}, \\
R \triangleright S= & \{(x, z) \in X \times Z \mid \\
& \forall y \in Y:(x, y) \in R \Leftarrow(y, z) \in S\}, \\
R \square S= & \{(x, z) \in X \times Z \mid \\
& \forall y \in Y:(x, y) \in R \Leftrightarrow(y, z) \in S\} .
\end{aligned}
$$

and are called basic (direct/circlet) composition, Bandler-Kohout (abbr. BK) subproduct, Bandler-Kohout superproduct and BandlerKohout square product, respectively.

The meaning of $(x, z) \in R \circ S$ is that a patient $x$ has at least one symptom belonging to a disease $z$ and therefore, it expresses a suspicion of having this disease, see Figure 1.

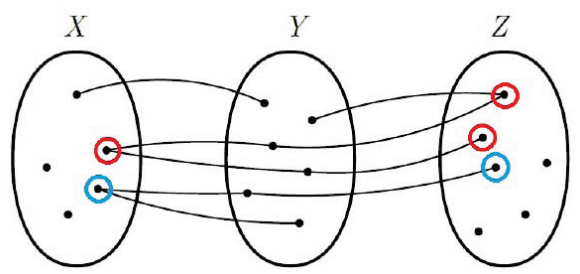

Figure 1: Graphical illustration of three universes and relations between their elements.

The "triangle" and square compositions (2)-(4) provide a sort of more accurate specification or a strengthening of the initial suspicion. The fact that $(x, z) \in R \triangleleft S$ means that all symptoms of a patient $x$ belong to a disease $z$, see Figure 2 . The fact that $(x, z) \in R \triangleright S$ means that a patient $x$ has all symptoms belonging to a disease $z$ (see Figure 3) and the meaning of $(x, z) \in R \square S$ is that a patient $x$ has all symptoms of the disease $z$ and all symptoms of the patient belong to a disease $z$, see Figure 4 .

Using the fact that the existential and universal quantifiers may be interpreted by the opera-

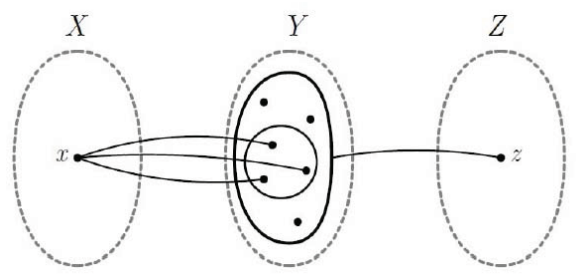

Figure 2: Graphical illustration of the BandlerKohout subproduct.

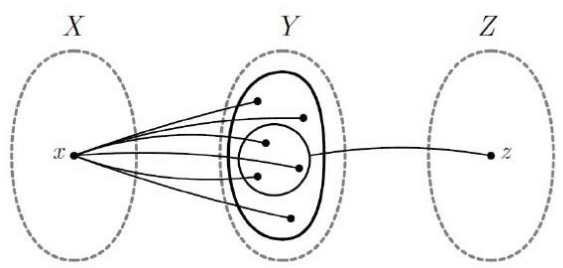

Figure 3: Graphical illustration of the BandlerKohout superproduct.

tions of supremum and infimum, respectively, formulas (1)-(4) may be rewritten into the following functional form:

$$
\begin{aligned}
\chi_{(R \circ S)}(x, z) & =\bigvee_{y \in Y}\left(\chi_{R}(x, y) \wedge \chi_{S}(y, z)\right), \\
\chi_{(R \triangleleft S)}(x, z) & =\bigwedge_{y \in Y}\left(\chi_{R}(x, y) \Rightarrow \chi_{S}(y, z)\right), \\
\chi_{(R \triangleright S)}(x, z) & =\bigwedge_{y \in Y}\left(\chi_{R}(x, y) \Leftarrow \chi_{S}(y, z)\right), \\
\chi_{(R \square S)}(x, z) & =\bigwedge_{y \in Y}\left(\chi_{R}(x, y) \Leftrightarrow \chi_{S}(y, z)\right),
\end{aligned}
$$

where $\chi_{R}, \chi_{S}$ and $\chi_{(R @ S)}$ denote characteristic functions of relations $R, S$ and $R @ S$ (for @ $\in\{\circ, \triangleleft, \triangleright, \square\})$, respectively. Furthermore, the symbol $\wedge$ denotes the minimum, the symbol $\Rightarrow$ expresses the binary operation of the classical implication and finally, the symbol $\Leftrightarrow$ denotes the operation of the classical equivalence.

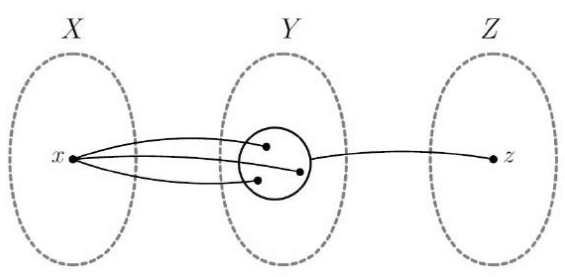

Figure 4: Graphical illustration of the BandlerKohout square product. 


\subsection{Fuzzy relational compositions}

Since usual symptoms such as high temperature, increased cholesterol or very high blood pressure are basically vaguely specified and imprecisely measured, and very often some symptoms do not clearly or necessarily belong to a given disease however, they might belong to it under some assumptions or conditions. Therefore, the extension of the compositions for fuzzy relations was highly desirable. Since such an extension causes that we deal with fuzzy relations which contain pairs of elements up to some degrees from the interval $[0,1]$, we have to take into account appropriate operations. Basically, it is appropriate to deal with a residuated lattice as the underlying algebraic structure, and the used operations will be left-continuous t-norms and their residual (bi-residual) implications [14]. Then, fuzzy relational compositions can be naturally defined based on the original definitions and using the operations from the residuated lattice, as introduced by Willis Bandler and Ladislav Kohout.

Let us fix a residuated lattice $\langle[0,1], \wedge, \vee, *, \rightarrow$ $0,1\rangle$ as the background algebraic structure for the rest of the paper. In other words, the multiplicative operation $*$ is a left-continuous t-norm and correspondingly the fuzzy implication $\rightarrow$ is a residual implication, and they both form an adjoint pair. Furthermore, let us fix the following notation for the set of all fuzzy sets on a given universe: $\mathcal{F}(U)=\{C \mid C: U \rightarrow[0,1]\}$.

Definition 1. Let $X, Y, Z$ be non-empty universes, and let $R \in \mathcal{F}(X \times Y), S \in \mathcal{F}(Y \times Z)$. Then, the compositions $\circ, \triangleleft, \triangleright, \square$ of fuzzy relations $R$ and $S$ are fuzzy relations on $X \times Z$ defined as follows:

$$
\begin{aligned}
& (R \circ S)(x, z)=\bigvee_{y \in Y}(R(x, y) * S(y, z)), \\
& (R \triangleleft S)(x, z)=\bigwedge_{y \in Y}(R(x, y) \rightarrow S(y, z)), \\
& (R \triangleright S)(x, z)=\bigwedge_{y \in Y}(R(x, y) \leftarrow S(y, z)), \\
& (R \square S)(x, z)=\bigwedge_{y \in Y}(R(x, y) \leftrightarrow S(y, z)),
\end{aligned}
$$

for all $x \in X$ and $z \in Z$.

Since $*$ is a t-norm, often denoted by a capital $\mathrm{T}$, the sup-* composition is also called sup$\mathrm{T}$ composition. Similarly, Bandler-Kohout products, since being constructed with help of the residual operation, are often called inf-R compositions.

Example 1. Let us consider the above mentioned medical diagnosis problem with $*$ and $\rightarrow$ to be the
Eukasiewicz operations and with fuzzy relations $R$ and $S$ given as follows

\begin{tabular}{c|c|c|c|c|}
$R$ & $y_{1}$ & $y_{2}$ & $y_{3}$ & $y_{4}$ \\
\hline$x_{1}$ & 0.9 & 1 & 0.8 & 0 \\
\hline$x_{2}$ & 0 & 0.9 & 0.8 & 0.1 \\
\hline$x_{3}$ & 0 & 0.8 & 0.9 & 0 \\
\hline$x_{4}$ & 0 & 0 & 1 & 0.9 \\
\hline
\end{tabular}

\begin{tabular}{c|c|c|c|c|c|}
$S$ & $z_{1}$ & $z_{2}$ & $z_{3}$ & $z_{4}$ & $z_{5}$ \\
\hline$y_{1}$ & 1 & 1 & 0.1 & 0.9 & 0 \\
\hline$y_{2}$ & 0.9 & 0.2 & 0.9 & 0 & 1 \\
\hline$y_{3}$ & 0 & 1 & 0 & 1 & 1 \\
\hline$y_{4}$ & 1 & 0 & 0.7 & 0.1 & 0.9 \\
\hline
\end{tabular}

If we compute the standard fuzzy relational compositions, e.g., the sup-* composition and the Bandler-Kohout square product:

\begin{tabular}{r|c|c|c|c|c|}
$R \circ S$ & $z_{1}$ & $z_{2}$ & $z_{3}$ & $z_{4}$ & $z_{5}$ \\
\hline$x_{1}$ & 0.9 & 0.9 & 0.9 & 0.8 & 1 \\
\hline$x_{2}$ & 0.8 & 0.8 & 0.8 & 0.8 & 0.9 \\
\hline$x_{3}$ & 0.7 & 0.9 & 0.7 & 0.9 & 0.9 \\
\hline$x_{4}$ & 0.9 & 1 & 0.6 & 1 & 1 \\
\hline
\end{tabular}

\begin{tabular}{r|c|c|c|c|c|}
$R \square S$ & $z_{1}$ & $z_{2}$ & $z_{3}$ & $z_{4}$ & $z_{5}$ \\
\hline$x_{1}$ & 0 & 0.2 & 0.2 & 0 & 0.1 \\
\hline$x_{2}$ & 0 & 0 & 0.2 & 0.1 & 0.2 \\
\hline$x_{3}$ & 0 & 0 & 0.1 & 0.1 & 0.1 \\
\hline$x_{4}$ & 0 & 0 & 0 & 0.1 & 0 \\
\hline
\end{tabular}

we will see, that while all patients are suspicious of having all diseases in a high degree when using $\circ$, if we want to strengthen the suspicion with help of $\square$, no patients is suspicious of having any disease in a high degree anymore.

From Example 1, one can easily conclude that the gap between the existence of a "connecting" symptom and the requirement for all such symptoms is too huge to capture all situations in practice. Therefore, strengthening the suspicion given by a simple existence by generalized quantifiers such as "Majority", "Many" or "A few", seem to be a natural step towards the higher practical applicability of the fuzzy relational compositions.

Remark 1. From Definition 1, one can note that for $x \in X$ such that $R(x, y)=0$ for all $y \in Y$, the composed relation $(R \triangleleft S)(x, z)=1$ for any $z \in Z$. In other words, if there is a patient with no symptoms, it is trivially true that for any given disease, all his symptoms are related to the given disease. Similarly, for $z \in Z$ such that $S(y, z)=0$ for all $y \in Y$, the composed relation $(R \triangleright S)(x, z)=1$ for all $x \in X$.

On the other hand, in such situations ( $R \circ$ $S)(x, z)=0$. So, the inf- $R$ compositions may hardly be viewed as strengthening of the suspicion determined by the sup-T composition if no suspicion was determined. De Baets and Kerre in [1] 
approached this problem by a redefinition of the original inf- $R$ compositions where an existence of joining element $y \in Y$ (symptom) is assumed. In this preliminary investigation we stay stuck to the original definitions and we leave the investigation of the later modification for further studies.

\section{Fuzzy relational compositions based on generalized quantifiers}

\subsection{Generalized quantifiers based on fuzzy measures}

We have recalled relational compositions and fuzzy relational compositions and introduced a motivation for an employment of generalized quantifiers. Particularly, for our goal, the so called monadic quantifiers of type $\langle 1\rangle$ determined by fuzzy measures [13] will be applied. First of all, let us recall some basic definitions.

Definition 2. Let $U=\left\{u_{1}, \ldots, u_{n}\right\}$ be a finite universe, let $\mathcal{P}(U)$ denote the power set of $U$, and let $\mu: \mathcal{P}(U) \rightarrow[0,1]$ be a normalized fuzzy measure, i.e., a monotone mapping with $\mu(\emptyset)=0$ and $\mu(U)=1$. We say that the fuzzy measure $\mu$ is invariant with respect to cardinality if the following condition holds:

$$
\forall A, B \in \mathcal{P}(U):|A|=|B| \Rightarrow \mu(A)=\mu(B)
$$

where $|\cdot|$ denotes the cardinality of a set.

Example 2. The measure called relative cardinality, given by

$$
\mu_{r c}(A)=\frac{|A|}{|U|},
$$

is invariant w.r.t. cardinality.

Example 3. Let $f:[0,1] \rightarrow[0,1]$ be a nondecreasing mapping with $f(0)=0$ and $f(1)=1$ then $\mu^{f}$ defined as $\mu^{f}(A)=f\left(\mu_{r c}(A)\right)$ is again a fuzzy measure that is invariant w.r.t. cardinality.

Note that all the models of linguistic evaluative expressions [15] of the type Big and modified by arbitrary linguistic hedge (e.g. More or less, Very, Roughly, Extremely etc.) are fuzzy sets on $[0,1]$ that fulfill the boundary conditions and thus, may be used in order to modify the original relative cardinality.

Another fuzzy measure that is being used frequently in the area of flexible query answering systems is the following.

Example 4. The following fuzzy measure

$$
\mu_{r c}^{1 / 2}(D)= \begin{cases}1 & \text { if } \mu_{r c}(D) \geq \frac{1}{2} \\ 0 & \text { otherwise. }\end{cases}
$$

Then $\mu_{r c}^{1 / 2}$ is a fuzzy measure on $U$ that is invariant w.r.t cardinality. It is used to construct a quantifier "at least half".

In this paper's framework, we will deal only with such fuzzy measures that are created by a modification of the relative cardinality by an appropriate fuzzy set (cf., Definition 3.7 in [13]).

Definition 3. Let $\mu$ be a fuzzy measure on $U$ that is invariant w.r.t. cardinality. A mapping $Q: \mathcal{F}(U) \rightarrow[0,1]$ defined by

$$
Q(C)=\bigvee_{D \in \mathcal{P}(U) \backslash\{\emptyset\}}\left(\left(\bigwedge_{u \in D} C(u)\right) * \mu(D)\right)
$$

is called (generalized/fuzzy) quantifier determined by fuzzy measure $\mu$.

Example 5. Let us consider the following fuzzy measures $\mu$

$$
\begin{aligned}
& \mu^{\forall}(D)= \begin{cases}1 & D \equiv U \\
0 & \text { otherwise, }\end{cases} \\
& \mu^{\exists}(D)= \begin{cases}0 & D \equiv \emptyset \\
1 & \text { otherwise. }\end{cases}
\end{aligned}
$$

Then the derived quantifiers are the classical universal and existential quantifiers.

One can immediately see that formula (9) is not very appropriate from the computational point of view as it requires calculation over all sets from $\mathcal{P}(U) \backslash\{\emptyset\}$. However, we may use the property of fuzzy measure being invariant w.r.t. cardinality and show that the fuzzy quantifier may be efficiently calculated.

Theorem 1. [12] Let $Q$ be a fuzzy quantifier on $U$ determined by a fuzzy measure $\mu$ that is invariant w.r.t. cardinality. Then,

$Q(C)=\bigvee_{i=1}^{n} C\left(u_{\pi(i)}\right) * \mu\left(\left\{u_{1}, \ldots, u_{i}\right\}\right), C \in \mathcal{F}(U)$

where $\pi$ is a permutation on $U$ such that $C\left(u_{\pi(1)}\right) \geq C\left(u_{\pi(2)}\right) \geq \cdots \geq C\left(u_{\pi(n)}\right)$.

The proof can be found in [12] as well and therefore, it is omitted.

Theorem 1 shows that the fuzzy quantifier defined by Definition 3 can be equivalently expressed by means of the Sugeno fuzzy integral. In other words, if we again apply the fuzzy measure $\mu^{f}=f\left(\mu_{r c}\right)$ that is constructed from the relative cardinality by some modifying fuzzy set $f$, then we get the following equality

$$
Q(C)=\bigvee_{i=1}^{n} C\left(u_{\pi(i)}\right) * f(i / n)
$$

which is very easy to be calculated. 
Remark 2. Note, that the use of Sugeno integrals for a construction of generalized quantifiers is rather standard especially in the area of flexible queries in relational databases [16, 10].

\subsection{Fuzzy relational compositions based on generalized quantifiers}

In this section, we follow [12] and directly apply the above introduced theory of generalized quantifiers to our problem of fuzzy relational compositions.

Using such modification, when the existential or the universal quantifier in the definition of the fuzzy relational compositions is replaced by the generalized one, and the previously provided theoretical apparatus, we obtain the following definition.

Definition 4. [12] Let $X, Y, Z$ be non-empty finite universes, let $R \in \mathcal{F}(X \times Y), S \in \mathcal{F}(Y \times Z)$. Let $\mu$ be a fuzzy measure on $Y$ that is invariant w.r.t. cardinality, and let $Q$ be a quantifier on $Y$ determined by the fuzzy measure $\mu$. Then, the $\diamond^{Q}, \triangleleft^{Q}, \triangleright^{Q}, \square^{Q}$ compositions of fuzzy relations $R$ and $S$ are fuzzy relations on $X \times Z$ defined as follows:

$\left(R \circ^{Q} S\right)(x, z)=$

$\bigvee_{D \in \mathcal{P}(Y) \backslash\{\emptyset\}}\left(\left(\bigwedge_{y \in D} R(x, y) * S(y, z)\right) * \mu(D)\right)$,

$\left(R \triangleleft^{Q} S\right)(x, z)=$

$\bigvee_{D \in \mathcal{P}(Y) \backslash\{\emptyset\}}\left(\left(\bigwedge_{y \in D} R(x, y) \rightarrow S(y, z)\right) * \mu(D)\right)$,

$\left(R \triangleright^{Q} S\right)(x, z)=$

$\bigvee_{D \in \mathcal{P}(Y) \backslash\{\emptyset\}}\left(\left(\bigwedge_{y \in D} R(x, y) \leftarrow S(y, z)\right) * \mu(D)\right)$, $\left(R \square^{Q} S\right)(x, z)=$

$\bigvee_{D \in \mathcal{P}(Y) \backslash\{\emptyset\}}\left(\left(\bigwedge_{y \in D} R(x, y) \leftrightarrow S(y, z)\right) * \mu(D)\right)$,

for all $x \in X$ and $z \in Z$.

Corollary 1. [12] Let $Q$ be a quantifier determined by a fuzzy measure that is modified from the relative cardinality by using function $f$. Then for all $x \in X$ and $z \in Z$ :

$$
\begin{aligned}
& \left(R \circ^{Q} S\right)(x, z)= \\
& \bigvee_{i=1}^{n}\left(\left(R\left(x, y_{\pi(i)}\right) * S\left(y_{\pi(i)}, z\right)\right) * f(i / n)\right),
\end{aligned}
$$

$$
\begin{aligned}
& \left(R \triangleleft^{Q} S\right)(x, z)= \\
& \bigvee_{i=1}^{n}\left(\left(R\left(x, y_{\pi(i)}\right) \rightarrow S\left(y_{\pi(i)}, z\right)\right) * f(i / n)\right), \\
& \left(R \triangleright^{Q} S\right)(x, z)= \\
& \bigvee_{i=1}^{n}\left(\left(R\left(x, y_{\pi(i)}\right) \leftarrow S\left(y_{\pi(i)}, z\right)\right) * f(i / n)\right), \\
& \left(R \square^{Q} S\right)(x, z)= \\
& \bigvee_{i=1}^{n}\left(\left(R\left(x, y_{\pi(i)}\right) \leftrightarrow S\left(y_{\pi(i)}, z\right)\right) * f(i / n)\right),
\end{aligned}
$$

where $\pi$ is a permutation such that $R\left(x, y_{\pi(i)}\right) \circledast$ $S\left(y_{\pi(i)}, z\right) \geq R\left(x, y_{\pi(i+1)}\right) \circledast S\left(y_{\pi(i+1)}, z\right)$, for $i=$ $1, \ldots, n-1$ and for $\circledast \in\{*, \rightarrow, \leftarrow, \leftrightarrow\}$.

It is easy to see that the original compositions are a special case of the newly defined compositions. Indeed, one may easily check that $R \circ S \equiv R \circ^{\exists} S$ and that $R \triangleleft S \equiv R \triangleleft^{\forall} S$, $R \triangleright S \equiv R \triangleright^{\forall} S$ and $R \square S \equiv R \square^{\forall} S$, using the fuzzy measures $\mu^{\forall}$ and $\mu^{\exists}$ provided in Example 5 .

Example 6. Let us consider fuzzy relations from Example 1 and assume again the Lukasiewicz operations. Furthermore, let us consider the fuzzy set modeling the meaning of the linguistic expression Quite Roughly Big (abbr. QRBi) which enables us to construct a generalized quantifier $Q=$ "Many". In a standard context, this fuzzy set takes values $\operatorname{QRBi}(1 / 4)=0, \operatorname{QRBi}(2 / 4)=$ $0, \operatorname{QRBi}(3 / 4)=0.86$ and $\operatorname{QRBi}(1)=1$. Then the newly suggested fuzzy relational composition $\square^{Q}$ gives the following results:

\begin{tabular}{r|c|c|c|c|c|}
$R \square^{Q} S$ & $z_{1}$ & $z_{2}$ & $z_{3}$ & $z_{4}$ & $z_{5}$ \\
\hline$x_{1}$ & 0.06 & 0.66 & 0.2 & 0.66 & 0.1 \\
\hline$x_{2}$ & 0 & 0.16 & 0.26 & 0.1 & 0.66 \\
\hline$x_{3}$ & 0 & 0.26 & 0.16 & 0.06 & 0.66 \\
\hline$x_{4}$ & 0 & 0 & 0 & 0.06 & 0.86 \\
\hline
\end{tabular}

As we may see, the composition $\square^{Q}$ strengthened the original suspicion given by o but did not strictly strengthen as by $\square$ which would require to have "All" symptoms of a given disease and to have "All" symptoms related a given disease. The composition $\square^{Q}$ requires only "Many" of the symptoms to have such properties and thus, better captures the natural vagueness and fuzziness of the real-world situation which leads to a strengthened suspicion of diseases $z_{5}$ (in the case of patients $x_{2}, x_{3}, x_{4}$ ) and of diseases $z_{2}, z_{4}$ (in the case of patient $x_{1}$ ).

\section{Properties}

There are many properties proved for original classical as well as fuzzy relational compositions. The question to be answered is whether 
the same or similar properties may be valid also for the compositions based on generalized quantifiers. As we will show, the answer is partly positive as some of the properties are preserved only in a weak form. In this section, we recall those properties of the newly defined compositions based on generalized quantifiers that were introduced in [12] and moreover, we provide readers with new properties determined during our recent investigation.

Theorem 2. [12] Let $X, Y, Z, U$ be finite universes, and let $R, R_{1}, R_{2} \in \mathcal{F}(X \times Y), S, S_{1}, S_{2} \in$ $\mathcal{F}(Y \times Z)$ and $T \in \mathcal{F}(Z \times U)$. Furthermore, let $\cup, \cap$ denote the Gödel union and intersection, respectively. Then,

1. $R \circ^{Q_{1}}\left(S \circ^{Q_{2}} T\right)=\left(R \circ^{Q_{1}} S\right) \circ^{Q_{2}} T$,

2. $R \square^{Q} S \subseteq\left(R \triangleleft^{Q} S\right) \cap\left(R \triangleright^{Q} S\right)$,

3. $R_{1} \subseteq R_{2} \Rightarrow\left(R_{1} \circ^{Q} S\right) \subseteq\left(R_{2} \circ^{Q} S\right)$ and $S_{1} \subseteq S_{2} \Rightarrow\left(R \circ^{Q} S_{1}\right) \subseteq\left(R \circ^{Q} S_{2}\right)$,

4. $R_{1} \subseteq R_{2} \Rightarrow\left(R_{1} \triangleleft^{Q} S\right) \supseteq\left(R_{2} \triangleleft^{Q} S\right)$ and $\left(R_{1} \triangleright^{Q} S\right) \subseteq\left(R_{2} \triangleright^{Q} S\right)$,

5. $\left(R_{1} \cup R_{2}\right) \circ^{Q} S \supseteq\left(R_{1} \circ^{Q} S\right) \cup\left(R_{2} \circ^{Q} S\right)$,

6. $\left(R_{1} \cap R_{2}\right) \triangleleft^{Q} S \supseteq\left(R_{1} \triangleleft^{Q} S\right) \cup\left(R_{2} \triangleleft^{Q} S\right)$,

7. $\left(R_{1} \cup R_{2}\right) \triangleright^{Q} S \supseteq\left(R_{1} \triangleright^{Q} S\right) \cup\left(R_{2} \triangleright^{Q} S\right)$,

8. $\left(R_{1} \cap R_{2}\right) \circ^{Q} S \subseteq\left(R_{1} \circ^{Q} S\right) \cap\left(R_{2} \circ^{Q} S\right)$,

9. $\left(R_{1} \cup R_{2}\right) \triangleleft^{Q} S \subseteq\left(R_{1} \triangleleft^{Q} S\right) \cap\left(R_{2} \triangleleft^{Q} S\right)$,

10. $\left(R_{1} \cap R_{2}\right) \triangleright^{Q} S \subseteq\left(R_{1} \triangleright^{Q} S\right) \cap\left(R_{2} \triangleright^{Q} S\right)$.

Sketch of the proof: All the properties are proved using the properties of background algebraic structure, i.e., using

$$
\begin{gathered}
(a \wedge b) * c=(a * c) \wedge(b * c), \\
(a \vee b) * c=(a * c) \vee(b * c), \\
(a \wedge b) \rightarrow c=(a \rightarrow c) \vee(b \rightarrow c), \\
(a \vee b) \rightarrow c=(a \rightarrow c) \wedge(b \rightarrow c), \\
a \rightarrow(b \wedge c)=(a \rightarrow b) \wedge(a \rightarrow c), \\
a \rightarrow(b \vee c)=(a \rightarrow b) \vee(a \rightarrow c), \\
\bigvee_{i}\left(\left(a_{i} * b\right) \wedge\left(a_{i} * c\right)\right) \leq \bigvee_{i}\left(a_{i} * b\right) \wedge \bigvee_{i}\left(a_{i} * c\right),
\end{gathered}
$$

and the antitonicity and the isotonicity of $\rightarrow$ in its first and second argument, respectively.

Remark 3. [12] Obviously, items 5.-10. may be also read as follows:

11. $R \circ^{Q}\left(S_{1} \cup S_{2}\right) \supseteq\left(R \circ^{Q} S_{1}\right) \cup\left(R \circ^{Q} S_{2}\right)$,

12. $R \triangleright^{Q}\left(S_{1} \cap S_{2}\right) \supseteq\left(R \triangleright^{Q} S_{1}\right) \cup\left(R \triangleright^{Q} S_{2}\right)$,

13. $R \triangleleft^{Q}\left(S_{1} \cup S_{2}\right) \supseteq\left(R \triangleleft^{Q} S_{1}\right) \cup\left(R \triangleleft^{Q} S_{2}\right)$,

14. $R \circ^{Q}\left(S_{1} \cap S_{2}\right) \subseteq\left(R \circ^{Q} S_{1}\right) \cap\left(R \circ^{Q} S_{2}\right)$,

15. $R \triangleright^{Q}\left(S_{1} \cup S_{2}\right) \subseteq\left(R \triangleright^{Q} S_{1}\right) \cap\left(R \triangleright^{Q} S_{2}\right)$,

16. $R \triangleleft^{Q}\left(S_{1} \cap S_{2}\right) \subseteq\left(R \triangleleft^{Q} S_{1}\right) \cap\left(R \triangleleft^{Q} S_{2}\right)$.

Remark 4. It should be noted, that Theorem 2 is provided in a modified form compared to [12]. In [12], the authors have introduced the Theorem with a mistake, particularly, items 5.-7 were mistakenly provided with equality instead of just an inclusion. This correction is of course also mirrored in the modification of Remark 3 that is also provided in a corrected form.

Theorem 3. Let $X, Y, Z, U$ be finite universes, and let $R \in \mathcal{F}(X \times Y), S \in \mathcal{F}(Y \times Z)$ and $T \in$ $\mathcal{F}(Z \times U)$. Then,

17. $R \circ^{Q_{1}}\left(S \triangleright^{Q_{2}} T\right) \subseteq\left(R \circ^{Q_{1}} S\right) \triangleright^{Q_{2}} T$, 18. $\left(R \triangleleft^{Q_{1}} S\right) \circ^{Q_{2}} T \subseteq R \triangleleft^{Q_{1}}\left(S \circ{ }^{Q_{2}} T\right)$.

Sketch of the proof: Again, by using the properties of the background residuated algebraic structure.

Theorem 4. Let $X, Y, Z$ be finite universes, and let $R \in \mathcal{F}(X \times Y), S \in \mathcal{F}(Y \times Z)$. Then,

19. $\left(R \circ^{Q} S\right)^{T}=S^{T} \circ^{Q} R^{T}$,

20. $\left(R \triangleleft^{Q} S\right)^{T}=S^{T} \triangleright^{Q} R^{T}$,

21. $\left(R \triangleright^{Q} S\right)^{T}=S^{T} \triangleleft^{Q} R^{T}$,

22. $\left(R \square^{Q} S\right)^{T}=S^{T} \square^{Q} R^{T}$.

where $R^{T}$ denotes the transposition of $R$, i.e., $R^{T}(x, y)=R(y, x)$.

Sketch of the proof: The proof is analogous to the proof for standard fuzzy relational compositions.

Lemma 1. Let $Q_{1}, Q_{2}$ be quantifiers determined by fuzzy measures $\mu_{1}, \mu_{2}$, respectively, such that $\mu_{1} \leq \mu_{2}$. Then,

23. $R \circ^{Q_{1}} S \subseteq R \circ^{Q_{2}} S$,

24. $R \triangleleft^{Q_{1}} S \subseteq R \triangleleft^{Q_{2}} S$,

25. $R \triangleright^{Q_{1}} S \subseteq R \triangleright^{Q_{2}} S$,

26. $R \square^{Q_{1}} S \subseteq R \square^{Q_{2}} S$.

Sketch of the proof: The proof is straightforward. It is sufficient to consider the monotonicity of $*$ and the antitonicity and the isotonicity of $\rightarrow$ in its first and second argument, respectively.

One can easily see that the measure $\mu^{\forall}$ is the smallest one, and the measure $\mu^{\exists}$ is the greatest one, which together with Lemma 1 provide many further properties of the compositions. For example, the following property holds for the standard compositions of fuzzy relational equations

$$
R \triangleleft(S \triangleleft T)=(R \circ S) \triangleleft T .
$$

Taking this into account jointly with Lemma 1, we can get

$$
\left(R \circ^{Q_{1}} S\right) \triangleleft T \subseteq(R \circ S) \triangleleft T=R \triangleleft(S \triangleleft T)
$$

and also

$$
R \triangleleft(S \triangleleft T) \subseteq R \triangleleft^{Q_{2}}(S \triangleleft T) \subseteq R \triangleleft^{Q_{2}}\left(S \triangleleft^{Q_{3}} T\right) .
$$

Thus, we can immediately formulate further valid property: 
27. $\left(R \circ^{Q_{1}} S\right) \triangleleft T \subseteq R \triangleleft^{Q_{2}}\left(S \triangleleft^{Q_{3}} T\right)$

for arbitrary quantifiers $Q_{1}, Q_{2}$ and $Q_{3}$.

Analogously, using the following facts

$$
\begin{aligned}
R \square(S \circ T) \subseteq R \triangleright(S \circ T)=(R \triangleright S) \triangleright T, \\
R \triangleleft(S \triangleright T)=(R \triangleleft S) \triangleright T
\end{aligned}
$$

and Lemma 1 we obtain the following properties:

28. $R \triangleright\left(S \circ^{Q_{1}} T\right) \subseteq\left(R \triangleright^{Q_{2}} S\right) \triangleright^{Q_{3}} T$,

29. $R \square\left(S \circ^{Q_{1}} T\right) \subseteq\left(R \triangleright^{Q_{2}} S\right) \triangleright^{Q_{3}} T$,

30. $R \triangleleft(S \triangleright T) \subseteq\left(R \triangleleft^{Q_{1}} S\right) \triangleright^{Q_{2}} T$,

31. $(R \triangleleft S) \triangleright T \subseteq R \triangleleft^{Q_{1}}\left(S \triangleright^{Q_{2}} T\right)$

for arbitrary quantifiers $Q_{1}, Q_{2}$ and $Q_{3}$.

\section{Images and preimages of fuzzy sets under fuzzy relations using generalized quantifiers}

Let us recall the concepts of image and preimage of a fuzzy set under a fuzzy relation.

Definition 5. Let $R \in \mathcal{F}(X \times Y)$, and let $A \in$ $\mathcal{F}(X)$. Then, the image of the fuzzy set $A$ under the fuzzy relation $R$ is a fuzzy set $R^{\varrho_{I}} A$ on $Y$ (for @ $\in\{\circ, \triangleleft, \triangleright, \square\}$ ) given by

$$
\begin{aligned}
& \left(R^{{ }^{I}} A\right)(y)=\bigvee_{x \in X}(A(x) * R(x, y)), \\
& \left(R^{\triangleleft_{I}} A\right)(y)=\bigwedge_{x \in X}(A(x) \rightarrow R(x, y)), \\
& \left(R^{\triangleright^{I}} A\right)(y)=\bigwedge_{x \in X}(A(x) \leftarrow R(x, y)), \\
& \left(R^{\square_{I}} A\right)(y)=\bigwedge_{x \in X}(A(x) \leftrightarrow R(x, y)),
\end{aligned}
$$

for all $y \in Y$.

Definition 6. Let $R \in \mathcal{F}(X \times Y)$, and let $B \in$ $\mathcal{F}(Y)$. Then, the preimage of the fuzzy set $B$ under the fuzzy relation $R$ is a fuzzy set $R^{\varrho_{P}} B$ on $X$ (for @ $\in\{\circ, \triangleleft, \triangleright, \square\}$ ) given by

$$
\begin{aligned}
& \left(R^{{ }^{P}} B\right)(x)=\bigvee_{y \in Y}(R(x, y) * B(y)), \\
& \left(R^{\triangleleft P} B\right)(x)=\bigwedge_{y \in Y}(R(x, y) \rightarrow B(y)), \\
& \left(R^{\triangleright^{P}} B\right)(x)=\bigwedge_{y \in Y}(R(x, y) \leftarrow B(y)), \\
& \left(R^{\square^{P}} B\right)(x)=\bigwedge_{y \in Y}(R(x, y) \leftrightarrow B(y)),
\end{aligned}
$$

for all $x \in X$.

Example 7. The meaning of the image as well as of the preimage can be illustrated on the obligatory example from medical diagnosis. Consider a fuzzy relation $R$ on the cartesian product of the set of symptoms $X$ and a set of patients $Y$. Let $A$ be a non-empty fuzzy set of special (chosen/searched) symptoms from $X$. Then, the images provide the following meanings:

- $R^{\circ}$ A is the fuzzy set of patients with at least one special symptom.

- $R^{\triangleleft I} A$ is the fuzzy set of patients with having all special symptoms.

- $R^{\triangleright_{I}} A$ is the fuzzy set of patients for whose all symptoms are among the special ones.

- $R^{\square_{I}} A$ is the fuzzy set of patients having all symptoms and no other symptoms.

The meaning of preimages could be described analogously on the medical diagnosis example. Here, the "query" sent to the database expressed by a fuzzy set $B \in \mathcal{F}(Y)$ of patients may have the meaning of, e.g., a fuzzy set of patients living in a neighborhood of some contamination source. Each patients belongs to the neighborhood up to a certain degree, therefore $B$ is a fuzzy set. The preimages then provide us with specific fuzzy sets of symptoms related to the fuzzy set of patients. The meaning can be determined analogously to the meaning of images and therefore, it is omitted. For further applications we refer e.g. to [17].

With an analogous motivation used for the introduction of fuzzy relational compositions based on generalized quantifiers, we propose the following definition of the images and preimages of fuzzy sets under fuzzy relations based on generalized quantifiers.

Definition 7. Let $R \in \mathcal{F}(X \times Y)$, let $A \in \mathcal{F}(X)$, and let $Q$ be a quantifier on $X$ determined by a fuzzy measure $\mu$ invariant w.r.t. cardinality. Then, the image of the fuzzy set $A$ under the fuzzy relation $R$ based on quantifier $Q$ is a fuzzy set $R_{I}^{@_{I}^{Q}} A$ on $Y$ (for @ $\in\{\circ, \triangleleft, \triangleright, \square\}$ ) given by

$$
\begin{aligned}
& \left(R^{\circ} A\right)(y)= \\
& \bigvee_{D \in \mathcal{P}(X) \backslash \emptyset}\left(\left(\bigwedge_{x \in D} A(x) * R(x, y)\right) * \mu(D)\right), \\
& \left(R^{\triangleleft_{I}^{Q}} A\right)(y)= \\
& \bigvee_{D \in \mathcal{P}(X) \backslash \emptyset}\left(\left(\bigwedge_{x \in D} A(x) \rightarrow R(x, y)\right) * \mu(D)\right), \\
& \left(R_{I}^{\triangleright} A\right)(y)= \\
& \bigvee_{D \in \mathcal{P}(X) \backslash \emptyset}\left(\left(\bigwedge_{x \in D} A(x) \leftarrow R(x, y)\right) * \mu(D)\right), \\
& \left(R^{\square_{I}^{Q}} A\right)(y)= \\
& \bigvee_{D \in \mathcal{P}(X) \backslash \emptyset}\left(\left(\bigwedge_{x \in D} A(x) \leftrightarrow R(x, y)\right) * \mu(D)\right)
\end{aligned}
$$


for all $y \in Y$.

Definition 8. Let $R \in \mathcal{F}(X \times Y)$, let $B \in \mathcal{F}(Y)$, and let $Q$ be a quantifier on $Y$ determined by a fuzzy measure $\mu$ invariant w.r.t. cardinality. Then, the preimage of the fuzzy set $B$ under the fuzzy relation $R$ based on quantifier $Q$ is a fuzzy set $R_{P}^{@ Q} B$ on $X$ (for $@ \in\{\circ, \triangleleft, \triangleright, \square\}$ ) given by

$$
\begin{aligned}
& \left(R^{\circ Q} B\right)(x)= \\
& \bigvee_{D \in \mathcal{P}(Y) \backslash \emptyset}\left(\left(\bigwedge_{y \in D} R(x, y) * B(y)\right) * \mu(D)\right) \\
& \left(R^{\left.\triangleleft P_{P}^{Q} B\right)(x)=}\right. \\
& \bigvee_{D \in \mathcal{P}(Y) \backslash \emptyset}\left(\left(\bigwedge_{y \in D} R(x, y) \rightarrow B(y)\right) * \mu(D)\right) \\
& \left(R^{\left.\triangleright{ }_{P}^{Q} B\right)(x)}=\right. \\
& \bigvee_{D \in \mathcal{P}(Y) \backslash \emptyset}\left(\left(\bigwedge_{y \in D} R(x, y) \leftarrow B(y)\right) * \mu(D)\right) \\
& \left.\left.\left(R^{\square} B\right)(x)=\bigwedge_{y \in D}^{Q} R(x, y) \leftrightarrow B(y)\right) * \mu(D)\right)
\end{aligned}
$$

for all $x \in X$.

Obviously, for computational purposes, we may use Theorem 1 similarly to the case of compositions of binary fuzzy relations.

We can identify an arbitrary fuzzy set $A$ with the binary fuzzy relation $\underline{A}$ using a dummy object $\underline{0}$ for the variable to be eliminated [2] as follows

$$
\underline{A}(x, y)= \begin{cases}A(x) & y=\underline{0} \\ 0 & \text { otherwise. }\end{cases}
$$

Due to the use of the dummy object, the images and preimages may be rewritten into the formal apparatus of compositions of fuzzy relations, namely:

$$
\begin{aligned}
& \left(R_{I}^{\circ_{I}^{Q}} A\right)(y)=\left(R^{T} \circ^{Q} \underline{A}\right)(y), \\
& \left(R^{\triangleleft_{I}^{Q}} A\right)(y)=\left(R^{T} \triangleright^{Q} \underline{A}\right)(y), \\
& \left(R^{\triangleright_{I}^{Q}} A\right)(y)=\left(R^{T} \triangleleft^{Q} \underline{A}\right)(y), \\
& \left(R^{\square_{I}^{Q}} A\right)(y)=\left(R^{T} \square^{Q} \underline{A}\right)(y), \\
& \left(R_{P}^{\circ_{P}^{Q}} B\right)(x)=\left(R \circ^{Q} \underline{B}\right)(x), \\
& \left(R^{\triangleleft_{P}^{Q}} B\right)(x)=\left(R \triangleleft^{Q} \underline{B}\right)(x), \\
& \left(R^{\triangleright_{P}^{Q}} B\right)(x)=\left(R \triangleright^{Q} \underline{B}\right)(x), \\
& \left(R^{\square_{P}^{Q}} B\right)(x)=\left(R \square^{Q} \underline{B}\right)(x) .
\end{aligned}
$$

This trick enables us to adopt all the results from the compositions, see Section 4. For example, using the associativity property 1 . from Theorem 2 and Theorem 4, one may easily derive new properties of images just by a simple equational calculus as follows:

$$
\begin{aligned}
& S^{\circ_{I}^{Q_{1}}}\left(R^{\circ_{I}^{Q_{2}}} A\right)=S^{T} \circ^{Q_{1}}\left(R^{T} \circ^{Q_{2}} \underline{A}\right) \\
& =\left(S^{T} \circ^{Q_{1}} R^{T}\right) \circ^{Q_{2}} \underline{A}=\left(R \circ^{Q_{1}} S\right)^{T} \circ^{Q_{2}} \underline{A}= \\
& \left(R \circ^{Q_{1}} S\right)^{\circ_{I}^{Q_{2}}} A .
\end{aligned}
$$

Thus, in a similar manner, we may easily prove all analogous properties of images and preimages to those of fuzzy relational compositions recalled and introduced in Section 4. All of them are formulated in the following corollary.

Corollary 2. Let $X, Y, Z$ be finite universes, let $A, A_{1}, A_{2} \in \mathcal{F}(X), B, B_{1}, B_{2} \in \mathcal{F}(Y)$, and $C \in$ $\mathcal{F}(Z)$, and let $R, R_{1}, R_{2} \in \mathcal{F}(X \times Y)$, and $S \in$ $\mathcal{F}(Y \times Z)$. Furthermore, let $\cup, \cap$ denote the Gödel union and intersection, respectively. Then,

27. $S_{{ }_{I}^{\circ}}^{Q_{1}}\left(R_{I}^{\circ_{2}^{Q_{2}}} A\right)=\left(R \circ \circ_{1}^{Q_{1}} S\right)^{\circ{ }_{I}^{Q_{2}}} A$,

28. $R_{P}^{\circ_{P}}\left(S_{P}^{\circ_{P}^{Q_{2}}} C\right)=\left(R \circ{ }^{Q_{1}} S\right)^{\circ}{ }_{P}^{Q_{2}} C$,

29. $R^{\square_{I}^{Q}} A \subseteq\left(R^{\triangleright} I_{I}^{Q} A\right) \cap\left(R^{\triangleleft_{I}^{Q} A}\right)$,

30. $R^{\square} B \subseteq\left(R_{P}^{\triangleleft_{P}^{Q}} B\right) \cap\left(R_{P}^{\triangleright} B\right)$,

31. $R_{1} \subseteq R_{2} \Rightarrow R_{1}^{\circ_{I}^{Q}} A \subseteq R_{2}^{\circ_{I}^{Q}} A$ and $B_{1} \subseteq A_{2} \Rightarrow R_{I}^{\circ Q} A_{1} \subseteq R_{I}^{\circ Q} A_{2}$,

32. $R_{1} \subseteq R_{2} \Rightarrow R_{1}^{\circ Q} B \subseteq R_{2}^{\circ P} B$ and

$B_{1} \subseteq B_{2} \Rightarrow R_{P}^{\circ Q} B_{1} \subseteq R_{P}^{\circ Q} B_{2}$,

33. $R_{1} \subseteq R_{2} \Rightarrow R_{1}^{\triangleleft_{I}^{Q}} A \subseteq R_{2}^{\triangleleft_{I}^{Q}} A$ and

$A_{1} \subseteq A_{2} \Rightarrow R^{\triangleleft_{I}^{Q}} A_{1} \supseteq R^{\triangleleft_{I}^{Q}} A_{2}$,

34. $R_{1} \subseteq R_{2} \Rightarrow R_{1}^{\triangleleft_{P}^{Q}} B \supseteq R_{2}^{\triangleleft_{P}^{Q}} B$ and

$B_{1} \subseteq B_{2} \Rightarrow R_{P}^{\triangleleft_{P}^{Q}} B_{1} \subseteq R_{P}^{\triangleleft_{P}^{Q}} B_{2}$,

35. $R_{1} \subseteq R_{2} \Rightarrow R_{1}^{\triangleright_{I}^{Q}} A \supseteq R_{2}^{\triangleright_{I}^{Q}} A$ and

$A_{1} \subseteq A_{2} \Rightarrow R_{I}^{\triangleright_{I}^{Q}} A_{1} \subseteq R_{I}^{\triangleright_{I}^{Q}} A_{2}$,

36. $R_{1} \subseteq R_{2} \Rightarrow R_{1}^{\triangleright^{Q}} B \subseteq R_{2}^{\triangleright^{Q}} B$ and

$B_{1} \subseteq B_{2} \Rightarrow R_{P}^{\triangleright} B_{1} \supseteq R_{P}^{\triangleright} B_{2}^{Q}$,

37. $\left(R_{1} \cup R_{2}\right)^{\circ Q}{ }_{I}^{Q} A \supseteq\left(R_{1}^{\circ Q} A\right) \cup\left(R_{2}^{\circ} A\right)$ and $R_{I}^{\circ Q}\left(A_{1} \cup A_{2}\right) \supseteq\left(R_{I}^{\circ Q} A_{1}\right) \cup\left(R_{I}^{\circ Q} A_{2}\right)$,

38. $\left(R_{1} \cup R_{2}\right)^{\circ}{ }_{P}^{Q} B \supseteq\left(R_{1}^{\circ Q} B\right) \cup\left(R_{2}^{\circ Q} B\right)$ and $R_{P}^{\circ Q}\left(B_{1} \cup B_{2}\right) \supseteq\left(R_{P}^{\circ Q} B_{1}\right) \cup\left(R_{P}^{\circ Q} B_{2}\right)$,

39. $\left(R_{1} \cap R_{2}\right)^{\circ Q} A \subseteq\left(R_{1}^{\circ Q} A\right) \cap\left(R_{2}^{\circ_{I}^{Q}} A\right)$ and $R_{I}^{\circ Q}\left(A_{1} \cap A_{2}\right) \subseteq\left(R_{P}^{\circ Q} A_{1}\right) \cap\left(R_{I}^{\circ_{I}^{Q}} A_{2}\right)$,

40. $\left(R_{1} \cap R_{2}\right)_{P}^{\circ Q} B \subseteq\left(R_{1}^{\circ Q} B\right) \cap\left(R_{2}^{\circ Q} B\right)$ and $R_{P}^{\circ Q}\left(B_{1} \cap B_{2}\right) \subseteq\left(R_{P}^{\circ Q} B_{1}\right) \cap\left(R_{P}^{\circ Q} B_{2}\right)$,

41. $\left(R_{1} \cap R_{2}\right) \triangleleft_{I}^{Q} A \subseteq\left(R_{1}^{\triangleleft_{I}^{Q}} A\right) \cap\left(R_{2}^{\triangleleft_{I}^{Q}} A\right)$ and $R^{\triangleleft_{I}^{Q}}\left(A_{1} \cap A_{2}\right) \supseteq\left(R^{\triangleleft_{I}^{Q}} A_{1}\right) \cup\left(R^{\triangleleft_{I}^{Q}} A_{2}\right)$,

42. $\left(R_{1} \cap R_{2}\right) \triangleleft_{P}^{Q} B \supseteq\left(R_{1}^{\triangleleft_{P}^{Q}} B\right) \cup\left(R_{2}^{\triangleleft_{P}^{Q}} B\right)$ and $R^{\triangleleft} \triangleleft_{P}^{Q}\left(B_{1} \cap B_{2}\right) \subseteq\left(R_{P}^{\triangleleft} B_{1}\right) \cap\left(R_{P}^{\triangleleft} B_{2}\right)$, 


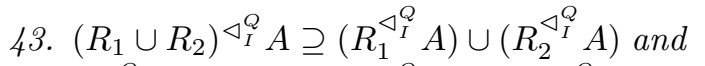
$R^{\triangleleft_{I}^{Q}}\left(A_{1} \cup A_{2}\right) \subseteq\left(R^{\triangleleft_{I}^{Q}} A_{1}\right) \cap\left(R^{\triangleleft_{I}^{Q}} A_{2}\right)$,

44. $\left(R_{1} \cup R_{2}\right)^{\triangleleft Q} B \subseteq\left(R_{1}^{\triangleleft P} B\right) \cap\left(R_{2}^{\triangleleft P} \triangleleft_{P}^{Q} B\right)$ and $R^{\triangleleft_{P}^{Q}}\left(B_{1} \cup B_{2}\right) \supseteq\left(R_{P}^{\triangleleft_{P}^{Q}} B_{1}\right) \cup\left(R_{P}^{\triangleleft_{P}^{Q}} B_{2}\right)$,

45. $\left(R_{1} \cup R_{2}\right)^{\triangleright_{I}^{Q}} A \subseteq\left(R_{1}^{\triangleright_{I}^{Q}} A\right) \cap\left(R_{2}^{\triangleright_{I}^{Q}} A\right)$ and $R_{I}^{\triangleright_{I}^{Q}}\left(A_{1} \cup A_{2}\right) \supseteq\left(R_{I}^{\triangleright_{I}^{Q}} A_{1}\right) \cup\left(R_{I}^{\triangleright_{I}^{Q}} A_{2}\right)$,

46. $\left(R_{1} \cup R_{2}\right)^{\triangleright_{P}^{Q}} B \supseteq\left(R_{1}^{\triangleright_{P}^{Q}} B\right) \cup\left(R_{2}^{\triangleright_{P}^{Q}} B\right)$ and $R_{P}^{\triangleright_{P}^{Q}}\left(B_{1} \cup B_{2}\right) \subseteq\left(R_{P}^{\triangleright_{P}^{Q}} B_{1}\right) \cap\left(R_{P}^{\triangleright_{P}^{Q}} B_{2}\right)$,

47. $\left(R_{1} \cap R_{2}\right)^{\triangleright_{I}^{Q}} A \supseteq\left(R_{1}^{\triangleright_{I}^{Q}} A\right) \cup\left(R_{2}^{\triangleright_{I}^{Q}} A\right)$ and $R_{I}^{\triangleright_{I}^{Q}}\left(A_{1} \cap A_{2}\right) \subseteq\left(R_{I}^{\triangleright_{I}^{Q}} A_{1}\right) \cap\left(R_{I}^{\triangleright_{I}^{Q}} A_{2}\right)$,

48. $\left(R_{1} \cap R_{2}\right)^{\triangleright_{P}^{Q}} B \subseteq\left(R_{1}^{\triangleright_{P}^{Q}} B\right) \cap\left(R_{2}^{\triangleright_{P}^{Q}} B\right)$ and $R_{P}^{\triangleright_{P}^{Q}}\left(B_{1} \cap B_{2}\right) \supseteq\left(R_{P}^{\triangleright_{P}^{Q}} B_{1}\right) \cup\left(R_{P}^{\triangleright P} B_{2}\right)$.

Sketch of the proof: The corollary exhaustively uses the above introduced equational calculus and the above proved properties of the compositions.

\section{Conclusions}

We have recalled classical and fuzzy relational compositions. Furthermore, we followed initial definition of compositions based on generalized quantifiers that is very common in fuzzy relational databases due to its potential to fill in a huge gap between compositions (or queries, in the database applications) based on the existential quantifier and the universal quantifier.

These quantifiers allow us to define fuzzy relational compositions with help of linguistically very natural quantifiers such as "A Few", "Many", "Majority" or "Most" and provide us with a wider choice for such fuzzy relational compositions that may better fit for each particular practical problem. We followed [12] in investigating the preservation of desirable properties that hold in the case of standard fuzzy relational compositions. An exhaustive list of properties is provided for compositions as well as of derived images and preimages. This gives a huge potential to employ the fuzzy relational compositions based on generalized quantifiers in many other areas of application, such as inference systems, where fuzzy relational compositions play a crucial role.

Fuzzy relation compositions have been defined using monadic quantifiers of type $\langle 1\rangle$ determined by fuzzy measures, it means that no restriction, e.g., on symptoms in Example 1, is assumed. Nevertheless, we can image situations when a restriction could be desirable, e.g., the importance of symptoms may vary in different countries. Therefore, it seems to be reasonable to extend fuzzy relation compositions using monadic quantifiers of type $\langle 1,1\rangle$ determined by fuzzy measures (see, e.g., $[18,19,20])$. The study of fuzzy relational compositions based on the type $\langle 1,1\rangle$ quantifiers is a subject of our future research.

\section{References}

[1] B. De Baets and E.E. Kerre. Fuzzy relational compositions. Fuzzy Sets and Systems, 60:109-120, 1993.

[2] L. Běhounek and M. Daňková. Relational compositions in fuzzy class theory. Fuzzy Sets and Systems, 160(8):1005-1036, 2009.

[3] L. A. Zadeh. Outline of a new approach to the analysis of complex systems and decision processes. IEEE Trans. Syst. Man Cybern., 3(1):28-44, 1973.

[4] W. Pedrycz. Applications of fuzzy relational equations for methods of reasoning in presence of fuzzy data. Fuzzy Sets and Systems, 16:163-175, 1985.

[5] M. Štěpnička and B. Jayaram. On the suitability of the Bandler-Kohout subproduct as an inference mechanism. IEEE Transactions on Fuzzy Systems, 18(2):285-298, 2010.

[6] M. Štěpnička, B. De Baets, and L. Nosková. Arithmetic fuzzy models. IEEE Transactions on Fuzzy Systems, 18:1058-1069, 2010.

[7] W. Bandler and L.J. Kohout. Semantics of implication operators and fuzzy relational products. International Journal of ManMachine Studies, 12(1):89 - 116, 1980.

[8] W. Bandler and L.J. Kohout. Relationalproduct architectures for information processing. Information Sciences, 37:25-37, 1985.

[9] D. Dubois and H. Prade. Semantics of quotient operators in fuzzy relational databases. Fuzzy Sets and Systems, 78:89-93, 1996.

[10] M. Delgado, D. Sanchez, and M. Amparo Vila. Fuzzy cardinality based evaluation of quantified sentences. International Journal of Approximate Reasoning, 23:23-66, 2000.

[11] O. Pivert and P. Bosc. Fuzzy preference queries to relational databases. Imperial College Press, London, 2012.

[12] M. Štěpnička and M. Holčapek. Fuzzy relational compositions based on generalized quantifiers. In Information Processing and Management of Uncertainty in KnowledgeBased Systems, PT II (IPMU'14), volume 443 of Communications in Computer and Information Science, pages 224-233. Springer, Berlin, 2014.

[13] A. Dvořák and M. Holčapek. L-fuzzy quantifiers of type $\langle 1\rangle$ determined by fuzzy measures. Fuzzy Sets and Systems, 160(23):3425-3452, 2009.

[14] V. Novák, I. Perfilieva, and J. Močkoř. Mathematical Principles of Fuzzy Logic. Kluwer 
Academic Publishers, Boston, 1999.

[15] V. Novák. A comprehensive theory of trichotomous evaluative linguistic expressions. Fuzzy Sets and Systems, 159(22):2939-2969, 2008.

[16] P. Bosc, L. Liétard, and O. Pivert. Sugeno fuzzy integral as a basis for the interpretation of flexible queries involving monotonic aggregates. Information Processing $\&$ Management, 39(2):287 - 306, 2003.

[17] D. Dubois, M. Nakata, and H. Prade. Find the items which certainly have (most of the) important characteristics to a sufficient degree. In Proc. 7th Conference of the International Fuzzy Systems Association (IFSA'97), volume 2, pages 243-248, Prague, Czech Republic, 1997.

[18] A. Dvořák and M. Holčapek. Type $\langle 1,1\rangle$ fuzzy quantifiers determined by fuzzy measures. Part I. Basic definitions and examples. Fuzzy Sets and Systems, 242:31-55, 2014.

[19] A. Dvořák and M. Holčapek. Type $\langle 1,1\rangle$ fuzzy quantifiers determined by fuzzy measures. Part II. Permutation and isomorphism invariances. Fuzzy Sets and Systems, 242:5688, 2014.

[20] A. Dvořák and M. Holčapek. Type $\langle 1,1\rangle$ fuzzy quantifiers determined by fuzzy measures. Part III. Extension, conservativity and extensionality. Fuzzy Sets and Systems (2015), in press. 